\title{
Polyaniline Hierarchical Structures Synthesized in Aqueous Solution: Micromats of
}

\section{Nanofibers}

Chuanqiang Zhou, Jie Han, Genping Song, Rong Guo*

School of Chemistry and Chemical Engineering, Yangzhou University, Yangzhou, 225002 Jiangsu, P. R. China.

E-mail address: guorong@yzu.edu.cn

\section{Supporting Information}

\section{Chemical Structure Characterization}

The chemical structures of micromats of PANI nanofibers were confirmed by Fourier-transform infrared (FTIR) spectroscope. The typical characteristic bands of PANI, such as $1580 \mathrm{~cm}^{-1}, 1504 \mathrm{~cm}^{-1}$, $1299 \mathrm{~cm}^{-1}$ and $1175 \mathrm{~cm}^{-1}$, are observed (Figure 2B). The result of XRD pattern of final micromats of PANI nanofibers shows unusual high crystallinity with main diffraction peaks centered at $2 \theta=18.5^{\circ}$, $23.3^{\circ}, 25.8^{\circ}, 26.3^{\circ}, 28.4^{\circ}$ (as exhibited in Figure $2 \mathrm{C}$ ), which is in accordance with the results reported. Besides, the doping/dedoping experiments were also conducted to see whether the PANI micromats can be reversibly doped and dedoped. The corresponding UV/vis spectra are shown in Figure S2. Doped PANI shows three characteristic absorption bands at 320, 448, and $830 \mathrm{~nm}$. After depoping with $0.1 \mathrm{M}$ $\mathrm{NH}_{4} \mathrm{OH}(\mathrm{aq})$, only two peaks at 370 and $546 \mathrm{~nm}$, which are assigned to the $\pi-\pi^{*}$ benzenoid transition and the benzenoid to quinoid excitotic transition, respectively, are observed. The UV/vis absorption spectra of PANI mat-like structures in the salt and base forms are consistent with previous results. 


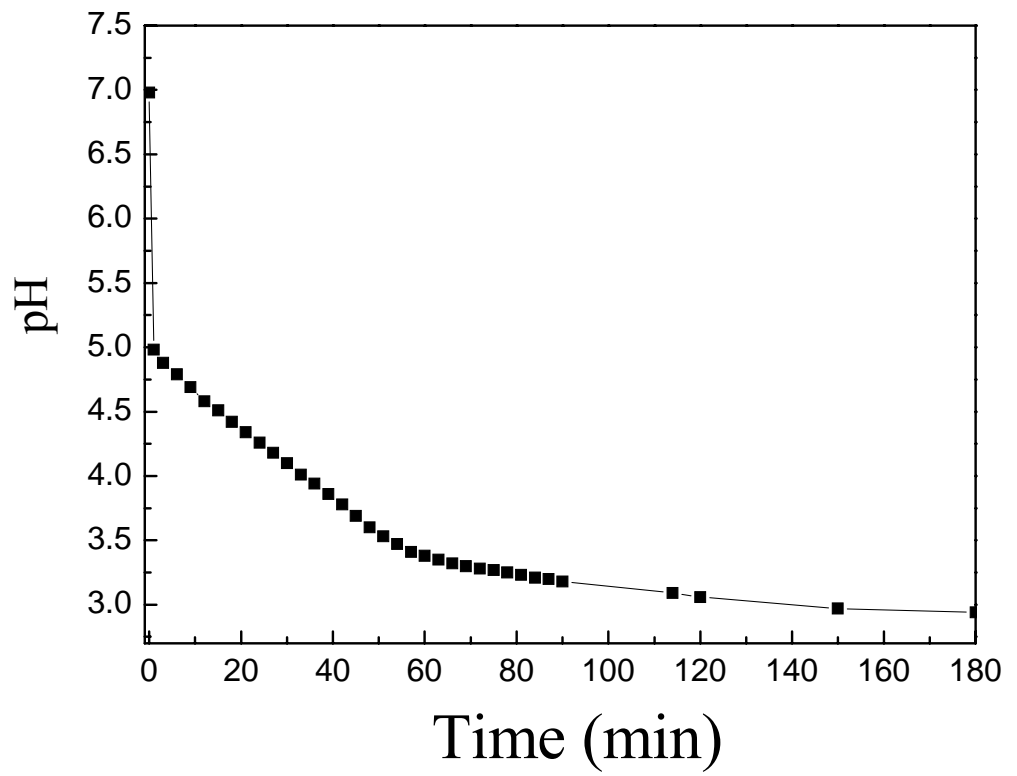

Figure S1. The $\mathrm{pH}$ value of solution decreasing as aniline polymerizes due to released protons. When the polymerization time is $1.5 \mathrm{~h}$, the $\mathrm{pH}$ value of solution decreases to 3.2 . 


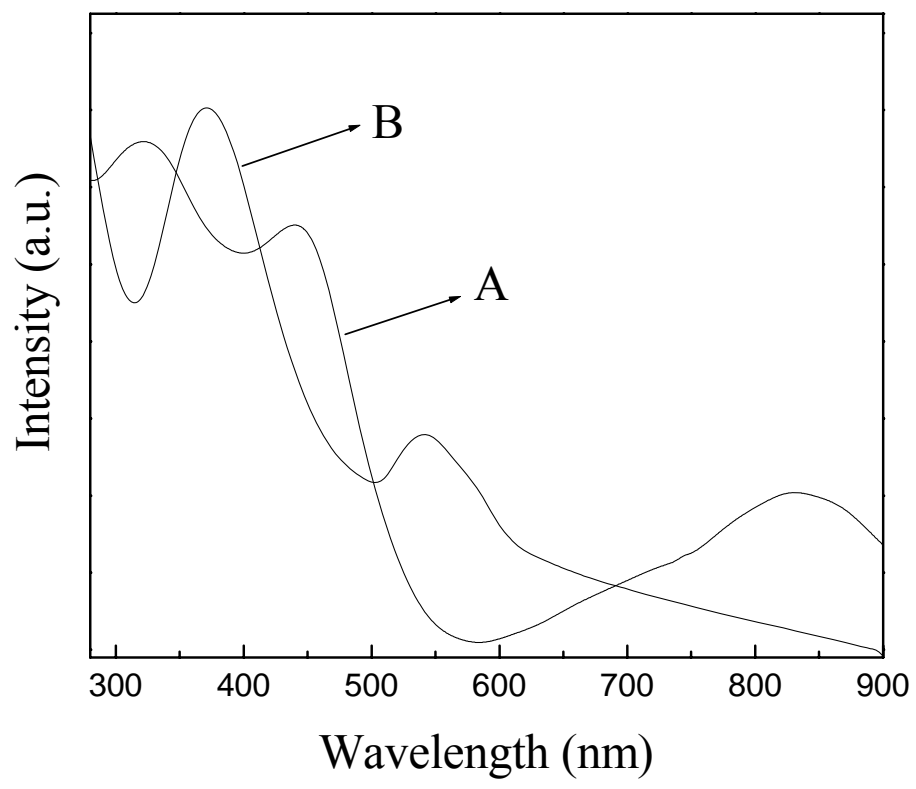

Figure S2. UV/vis spectra of PANI micromats after (A) doping with $1 \mathrm{M} \mathrm{HCl}(\mathrm{aq})$ and (B) dedoping with $0.1 \mathrm{M} \mathrm{NH}_{4} \mathrm{OH}$ (aq). 